\title{
Risco cardiovascular aumentado e o papel da síndrome metabólica em idosos hipertensos
}

\author{
Increased cardiovascular risk and role of metabolic syndrome in hypertensive elderly
}

Aumento del riesgo cardiovascular y el papel del síndrome metabólico en personas hipertensas de la

tercera edad

Manoela Vieira Gomes da $\operatorname{Costa}^{1}$ (1) Luciano Ramos de Lima $^{2}$ (1)

Izabel Cristina Rodrigues da Silva ${ }^{1}$ (1) Tania Cristina Morais Santa Barbara Rehem ${ }^{3}$ (1) Silvana Schwerz Funghetto ${ }^{1}$ (I) Marina Morato Stival ${ }^{1}$ (D)

1. Universidade de Brasília, Faculdade de Ceilândia, Programa de Pós-Graduação Ciências e Tecnologias em Saúde. Brasília, DF, Brasil.

2. Universidade de Brasília, Faculdade de Ceilândia. Brasília, DF, Brasil.

3. Universidade de Brasília, Faculdade de Saúde, Programa de Pós-Graduação em Enfermagem. Brasília, DF, Brasil.

\begin{abstract}
RESUMO
Objetivo: avaliar a síndrome metabólica e o risco cardiovascular de idosos hipertensos atendidos na atenção primária. Métodos: estudo transversal realizado com 154 idosos hipertensos de uma Unidade Básica de Saúde do Distrito Federal. Um instrumento estruturado investigou o perfil dos idosos. Para a classificação da síndrome metabólica, consideraram-se os critérios propostos pela National Cholesterol Education Program - Adult Treatment Panel III. Para análise do risco cardiovascular, utilizou-se o escore de risco de Framingham. Foi realizada análise estatística e inferencial com a utilização da ANOVA, teste qui-quadrado e exato de Fisher, além da odds ratio e seu intervalo de confiança de 95\% para estimar o risco cardiovascular entre os grupos. Resultados: $64,9 \%$ dos idosos hipertensos eram obesos. Síndrome metabólica foi evidenciada em 70,8\%. Observou-se que $27,2 \%$ apresentaram baixo, $46,8 \%$ moderado e $26,0 \%$ elevado risco cardiovascular, sendo que o sexo feminino e a idade avançada influenciaram negativamente o risco. Idosos com síndrome metabólica apresentaram 7,19 vezes mais chances de terem elevado risco cardiovascular. Considerações finais e implicações para a prática: os idosos hipertensos apresentaram uma elevada prevalência de síndrome metabólica que aumentou significativamente o risco cardiovascular. Este resultado possibilita um melhor planejamento da assistência de enfermagem pelo enfermeiro da atenção primária à saúde.
\end{abstract}

Palavras-chave: Idoso; Hipertensão; Síndrome Metabólica; Doenças Cardiovasculares.

\begin{abstract}
Objective: To evaluate metabolic syndrome and cardiovascular risk for hypertensive elderly patients treated in primary care. Methods: A cross-sectional study carried out with 154 hypertensive elderly from a Basic Health Unit in the Federal District. A structured instrument investigated the profile of the elderly. For classifying the metabolic syndrome, the criteria proposed by the National Cholesterol Education Program - Treatment Panel for Adults III were considered. For cardiovascular risk analysis, the Framingham risk score was used. Statistical and inferential analysis was performed using ANOVA, chi-square test and Fisher's exact test, in addition to Odds Ratio and its $95 \%$ confidence interval to estimate cardiovascular risk among the groups. Results: $64.9 \%$ of the hypertensive elderly were obese. Metabolic syndrome was evidenced in $70.8 \%$. It was noted that $27.2 \%$ had low, $46.8 \%$ moderate, and $26.0 \%$ high cardiovascular risk, and that being a woman and of advanced aged negatively influenced the risk. Older adults with metabolic syndrome showed 7.19 times more likelihood to have high cardiovascular risk. Final considerations and implications for the practice: The hypertensive elderly patients had high metabolic syndrome prevalence, which significantly increased cardiovascular risk. This result allows for a better planning of nursing care by the nurses in primary health care.
\end{abstract}

Keywords: Elderly; Hypertension; Metabolic Syndrome; Cardiovascular Diseases.

\section{RESUMEN}

Objetivo: evaluar el síndrome metabólico y el riesgo cardiovascular de pacientes hipertensos de la tercera edad tratados en la atención primaria. Métodos: estudio transversal realizado con 154 personas de la tercera edad hipertensas de una Unidad Básica de Salud del Distrito Federal. Se investigó el perfil de estas personas a través de un instrumento estructurado. Para clasificar el síndrome metabólico se utilizaron los criterios propuestos por el National Cholesterol Education Program - Adult Treatment Panel III. Para analizar el riesgo cardiovascular, se utilizó el escore de riesgo de Framingham. El análisis estadístico e inferencial se realizó mediante ANOVA, chi-cuadrado y la prueba exacta de Fisher, además de la odds ratio y su intervalo de confianza del $95 \%$ para estimar el riesgo cardiovascular entre los grupos. Resultados: el 64,9\% de las personas hipertensas de la tercera edad eran obesas. El síndrome metabólico se observó en el $70,8 \%$ de los casos. Se pudo observar que el $27,2 \%$ eran de riesgo cardiovascular bajo, $46,8 \%$ moderado y $26,0 \%$ alto, siendo que el sexo femenino y la edad avanzada influencian el aumento del riesgo. Las personas mayores de edad poseen 7,19 veces más probabilidades de tener un alto riesgo cardiovascular. Consideraciones finales e implicaciones para la práctica: los pacientes hipertensos de edad avanzada tenían una prevalencia elevada de síndrome metabólico que aumentaba, considerablemente, el riesgo cardiovascular. Este resultado permite una mejor planificación de la atención de enfermería por parte de las enfermeras en la atención primaria de la salud.

Palabras-clave: Anciano; Hipertensión; Síndrome Metabólico; Enfermedades Cardiovasculares.
Recebido em 09/03/2020.

Aprovado em 22/05/2020.

DOI:

https://doi.org/10.1590/2177-9465-EAN-2020-0055 


\section{INTRODUÇÃO}

A hipertensão arterial sistêmica (HAS) é uma condição clínica multifatorial caracterizada por elevação sustentada dos níveis pressóricos $\geq 140$ e/ou $90 \mathrm{mmHg} .{ }^{1}$ É a doença crônica não transmissível (DCNT) mais predominante entre os idosos, uma vez que sua prevalência aumenta progressivamente com o envelhecimento. ${ }^{2}$

Entre os idosos brasileiros, em 2013, apenas 22,3\% declararam não apresentar nenhuma DCNT. Aproximadamente a metade, $48,6 \%$, declarou ter uma ou duas, e $29,1 \%$, três ou mais doenças. Nesta população, a HAS vem acompanhada por modificações no perfil nutricional, pois se tem observado um aumento da obesidade e de outras DCNT. ${ }^{3}$

Esse cenário vem acarretando alterações no estado de saúde dos idosos e, consequentemente, maior exposição aos fatores de risco relacionados à síndrome metabólica (SM), que representa um grupo de fatores de risco cardiometabólicos que incluem obesidade abdominal combinada com elevação de pressão arterial, glicemia de jejum e triglicerídeos, além de redução do nível de lipoproteína de alta densidade (HDL). ${ }^{4} \mathrm{~A}$ SM tem despertado atenção da comunidade científica, não somente pelo impacto dos respectivos componentes, mas, principalmente, pela elevada prevalência dos fatores de risco cardiovasculares. ${ }^{5}$

Estudos realizados com idosos no Brasil observaram prevalências de SM que variaram entre 20 e $60 \%$. $^{6-10} \mathrm{O}$ envelhecimento é considerado um fator de risco para a maioria das doenças cardiovasculares, assim como de inúmeras comorbidades, tornando os idosos o grupo etário de maior heterogeneidade e complexidade. Por isso, a SM tem sido relacionada aos eventos cardiovasculares em idosos. ${ }^{11-13}$

Neste sentido, alguns instrumentos estão disponíveis para a avaliação do risco cardiovascular (RCV). Cabe ressaltar o escore de risco de Framingham (ERF) que é uma ferramenta útil e de fácil aplicação no cotidiano. Classifica os indivíduos em baixo, moderado e elevado risco de desenvolver uma doença cardiovascular nos próximos dez anos, por meio de uma pontuação que auxilia na definição de condutas terapêuticas. ${ }^{2}$

Poucos estudos realizados no Brasil, com idosos hipertensos, avaliou a relação existente entre a SM e RCV, avaliada pelo Framingham. A nível internacional, alguns estudos investigaram essa relação. ${ }^{13,14}$ Sabe-se que intervenções no estilo de vida são estratégias importantes para a prevenção e o tratamento da SM.1,4

Nessa direção, considera-se a importância de realização de estudos que fundamentem a avaliação e o planejamento do cuidado realizado pelo enfermeiro, principalmente no cenário da atenção primária à saúde, local de maior atendimento do idoso com DCNT. Diante do exposto, este estudo objetiva avaliar a síndrome metabólica e o risco cardiovascular de idosos hipertensos atendidos na atenção primária.

\section{MÉTODO}

Trata-se de um estudo transversal, de abordagem quantitativa, realizado com idosos usuários de uma Unidade Básica de Saúde (UBS) do Distrito Federal, no período de julho a agosto de 2019.
No cálculo do tamanho amostral, foram considerados os seguintes parâmetros estatísticos: nível de confiança de 95\% e erro estatístico de 5\%. Para o cálculo, foi considerado o total de idosos cadastrados no Grupo de Hipertensos da UBS ( $\mathrm{N}=300)$. A amostragem foi aleatória, mediante sorteio realizado de acordo com o número de cadastro do idoso no referido grupo. A amostra inicial foi composta por 160 idosos, de ambos os sexos, que atenderam aos seguintes critérios de inclusão: idade maior ou igual a 60 anos, com diagnóstico médico de hipertensão arterial sistêmica há mais de seis meses. Foram excluídos 6 idosos que não completaram a coleta de dados, finalizando a amostra em 154 idosos.

O idoso foi orientado a comparecer em jejum de 12 horas na unidade, em dia e horário agendados previamente. A coleta de sangue foi realizada por meio da punção venosa, preferencialmente na fossa antecubital, para dosagem das concentrações de triglicerídeos (TG), lipoproteína de alta densidade (HDL), lipoproteína de baixa densidade (LDL), colesterol total (COL), glicemia de jejum (GLI), hemoglobina glicada ( $\mathrm{HbA} 1 \mathrm{c})$ e hematócrito $(\mathrm{HT})$. Os exames bioquímicos foram realizados em um laboratório de análises clínicas do Distrito Federal, financiados pelo orçamento do projeto de pesquisa.

Um instrumento estruturado foi utilizado para investigar os dados demográficos (sexo e idade), hábitos de vida (tabagismo, etilismo, sedentarismo e sono), número de medicações utilizadas e presença de DM (autorreferida). Posteriormente, procedeu-se à aferição da pressão arterial (PA), que foi realizada seguindo todas as etapas preconizadas na VII Diretrizes Brasileiras de Hipertensão Arterial. A pressão arterial foi medida pela técnica auscultatória, com esfigmomanômetro de calibrado, com manguito adaptado no braço esquerdo do paciente e estetoscópio posicionado sobre a linha da artéria braquial. ${ }^{1}$

Para avaliação das variáveis antropométricas, foram mensurados peso, estatura e circunferência abdominal (CA). No momento da mensuração, os idosos foram pesados com roupas leves e descalços. Foi utilizada uma balança portátil com capacidade de $150 \mathrm{~kg}$ e sensibilidade de $100 \mathrm{~g}$ (Plenna ${ }^{\circledR}$ ). Para a verificação da estatura, foi utilizado um estadiômetro portátil Sanny ${ }^{\circledR}$, com altura máxima de $2,05 \mathrm{~m}$. Para isso, os idosos foram medidos na posição ereta, com as mãos lateralizadas ao corpo, a cabeça alinhada à linha do horizonte e com os calcanhares alinhados. A medida da CA foi realizada em pé, com fita métrica inelástica (Sanny, com $200 \mathrm{~cm}$, divisão de $1 \mathrm{~mm}$ ) que circundou a linha natural da cintura, na região mais estreita entre o tórax e o quadril, acima da cicatriz umbilical. ${ }^{15}$

O índice de massa corporal (IMC) foi calculado considerando o peso (em quilogramas) dividido pela altura (em metro) ao quadrado, sendo classificado em normal $\left(18,5 \mathrm{~kg} / \mathrm{m}^{2}\right.$ a $\left.24,9 \mathrm{~kg} / \mathrm{m}^{2}\right)$, sobrepeso $\left(25 \mathrm{~kg} / \mathrm{m}^{2}\right.$ a $\left.29,9 \mathrm{~kg} / \mathrm{m}^{2}\right)$ e obesidade $\left(\geq 30 \mathrm{~kg} / \mathrm{m}^{2}\right){ }^{3}$

Para a classificação da SM, consideraram-se os critérios propostos pela National Cholesterol Education Program - Adult Treatment Panel III (NCEP-ATP III), quando três dos cinco fatores apresentados estavam presentes: 1) TG $\geq 150 \mathrm{mg} / \mathrm{dL}$ ou uso de medicamentos para dislipidemia; 2) PAS $\geq 130 \mathrm{mmHg}$, 
$P A D \geq 85 \mathrm{mmHg}$, ou o uso de medicamentos anti-hipertensivos; 3) $\mathrm{GLI} \geq 110 \mathrm{mg} / \mathrm{dL}$ ou uso de medicamentos para DM; 4) $\mathrm{HDL}<40 \mathrm{mg} / \mathrm{dL}$ (homem) ou $<50 \mathrm{mg} / \mathrm{dL}$ (mulher) ou uso de medicamentos para dislipidemia; e 5) $\mathrm{CA} \geq 88 \mathrm{~cm}$ (mulher) e $\geq 102 \mathrm{~cm}$ (homem). ${ }^{4}$

Para análise do RCV, utilizou-se o ERF, no qual cada variável apresenta valores que possuem pontuações específicas, sendo positivas ou negativas. A pontuação total do escore considera as seguintes variáveis: sexo, idade, tabagismo, DM, HDL, LDL, PAS e PAD. O escore obtido corresponde a um percentual de probabilidade de ocorrência de doença arterial coronariana nos próximos 10 anos. Os idosos foram classificados nas seguintes categorias: baixo RCV <10\%; moderado RCV entre 10 e $20 \%$; e elevado RCV $>20 \%$ de ter um evento cardiovascular nos próximos dez anos. $^{2}$

Os dados foram analisados no software Statistical Package for the Social Sciences (SPSS), versão 20.0. Inicialmente, foram calculadas medidas descritivas. Na análise da normalidade das variáveis foi adotado o teste de Kolmogorov Smirnov. A descrição das variáveis contínuas foi por meio de médias e desvios padrão. A ANOVA com ajuste de Bonferroni foi empregada objetivando comparar diferenças entre as médias das variáveis avaliadas. Para verificação das diferenças entre as proporções, foi utilizado o teste Qui-Quadrado e o teste exato de Fisher. O odds ratio e seu intervalo de confiança de $95 \%$ foram calculados para estimar o RCV entre os grupos. Foi adotado um nível de significância de $5 \%$ para todos os testes.
Esta pesquisa faz parte do projeto "Abordagem das condições crônicas não transmissíveis na atenção primária à saúde" do Grupo de Pesquisa Saúde, Cuidado e Envelhecimento da Faculdade de Ceilândia da Universidade de Brasília (FCE/UnB), que foi submetido ao Comitê de Ética em Pesquisa (CEP) da Secretaria de Saúde do Distrito Federal (SES/DF) e aprovado com parecer de número 1.355.211 e CAAE 50367215.5.0000.5553. Todos os participantes aceitaram participar da pesquisa e assinaram o Termo de Consentimento Livre e Esclarecido.

\section{RESULTADOS}

Dos 154 idosos hipertensos, a maioria era do sexo feminino $(81,2 \%)$ e com média de idade de 68,1 $\pm 6,2$ anos. Em relação ao ERF, observou-se que $27,2 \%$ apresentaram baixo, $46,8 \%$ moderado e $26,0 \%$ elevado RCV. Houve maior prevalência do sexo feminino naqueles com baixo e moderado RCV $(p=0,004)$ e verificou-se que a média de idade aumenta de acordo com a gravidade do RCV ( $p=0,037)$ (Tabela 1).

No que tange às variáveis clínicas e bioquímicas, observou-se que o IMC, PAS, PAD, COL, TG, LDL, HDL, GLI, HbA1c, HT e a presença do DM apresentaram diferenças estatísticas, uma vez que os idosos hipertensos com elevado RCV apresentaram piores resultados (Tabela 1).

Ao analisar os hábitos de vida dos idosos hipertensos, ficou evidente que o sedentarismo influenciou negativamente no RCV $(p=0,000)$. Cabe ressaltar que apesar de não apresentarem

Tabela 1. Variáveis demográficas, antropométricas, bioquímicas e clínicas de acordo com o risco cardiovascular de Framingham dos idosos. Brasília, 2019. (n=154).

\begin{tabular}{lcccc}
\hline \multicolumn{1}{c}{ Parâmetros } & $\begin{array}{c}\text { Baixo risco } \\
(\mathrm{n}=42)\end{array}$ & $\begin{array}{c}\text { Moderado risco } \\
(\mathrm{n}=72)\end{array}$ & $\begin{array}{c}\text { Elevado risco } \\
(\mathrm{n}=40)\end{array}$ & Valor P \\
\hline Sexo (feminino/masculino) & $95,2 / 4,8$ & $80,6 / 19,4$ & $65,8 / 34,2$ & $0,004^{* *}$ \\
\hline Idade (anos) & $66 \pm 5$ & $68 \pm 6$ & $70 \pm 7$ & $0,037^{*}$ \\
\hline IMC (kg/cm $\left.{ }^{2}\right)$ & $30 \pm 5$ & $31 \pm 5$ & $33 \pm 5$ & $0,042^{*}$ \\
\hline CA (cm) & $98 \pm 11$ & $99 \pm 12$ & $103 \pm 12$ & 0,078 \\
\hline PAS (mmHg) & $134 \pm 19$ & $132 \pm 16$ & $152 \pm 18$ & $0,000^{*}$ \\
\hline PAD (mmHg) & $79 \pm 14$ & $79 \pm 12$ & $89 \pm 12$ & $0,000^{*}$ \\
COL (mmol/L) & $183,7 \pm 40,0$ & $187,3 \pm 44,0$ & $214,8 \pm 39,1$ & $0,001^{*}$ \\
\hline TG (mmol/L) & $131,2 \pm 52,0$ & $155,5 \pm 76,0$ & $186,9 \pm 97,5$ & $0,004^{*}$ \\
\hline LDL (mmol/L) & $105,6 \pm 39,2$ & $109,4 \pm 38,4$ & $132,0 \pm 35,4$ & $0,011^{*}$ \\
\hline HDL (mmol/L) & $51,8 \pm 6,3$ & $47,5 \pm 9,5$ & $43,5 \pm 8,2$ & $0,000^{*}$ \\
\hline GLI (mmol/L) & $112,7 \pm 54,0$ & $113,0 \pm 38,2$ & $152,5 \pm 71,5$ & $0,001^{*}$ \\
\hline HbA1c (\%) & $6,0 \pm 1,3$ & $6,2 \pm 1,3$ & $7,3 \pm 2,0$ & $0,000^{*}$ \\
\hline HT (\%) & $42,7 \pm 3,2$ & $42,0 \pm 3,9$ & $44,6 \pm 3,5$ & $0,001^{*}$ \\
\hline DM (\%) & 38,1 & 59,7 & 84,2 & $0,000^{* *}$ \\
\hline
\end{tabular}

*ANOVA com ajuste de Bonferroni; **Teste exato de Fisher. IMC: índice de massa corporal; CA: circunferência abdominal; PGC: percentual de gordura corporal; PAS: pressão arterial sistólica; PAD: pressão arterial diastólica; COL: colesterol; TG: triglicerídeos; LDL: lipoproteína de baixa densidade; HDL: lipoproteína de alta densidade; GLI: glicemia; HbA1c: hemoglobina glicada; HT: hematócrito; DM: diabetes mellitus. 
significância estatística, uma maior prevalência de tabagismo e etilismo foi observada no grupo de idosos com elevado RCV (Tabela 2).

De acordo com o estado nutricional, $10,4 \%$ dos idosos hipertensos eram eutróficos, $24,7 \%$ com sobrepeso e $64,9 \%$ obesos. A média do ERF foi de 11,8 $\$ 3,0$ e ao relacioná-la com o estado nutricional, de acordo com a presença ou não de SM, foi evidenciado que os idosos com SM apresentaram maiores medianas de ERF, quando comparados aqueles sem SM $(p=0,000)$. Além disso, observaram-se maiores $E R F$ nos idosos com SM e obesos (ERF=8 a 20\%) (Figura 1).
A SM foi evidenciada em $70,8 \%$ dos idosos hipertensos, sendo que $32,5 \%$ apresentaram três componentes, $26,0 \%$ apresentaram quatro e $12,3 \%$ apresentaram cinco componentes. A prevalência de SM foi significativamente maior no grupo de idosos com elevado RCV, pois nos idosos com SM foi identificado um risco 7,19 vezes maior de apresentarem elevado RCV. Em relação ao número de componentes da SM, observou-se que aqueles com quatro e cinco componentes demonstraram, respectivamente, 4,01 e 3,74 vezes mais chances de terem elevado RCV (Tabela 3).

Tabela 2. Hábitos de vida e número de medicamentos de acordo com o risco cardiovascular de Framingham dos idosos. Brasília, 2019. $(n=154)$.

\begin{tabular}{lcccl}
\hline & $\begin{array}{c}\text { Baixo risco } \\
(\mathrm{n}=42)\end{array}$ & $\begin{array}{c}\text { Moderado risco } \\
(\mathrm{n}=72)\end{array}$ & $\begin{array}{c}\text { Elevado risco } \\
(\mathrm{n}=40)\end{array}$ & Valor P \\
\hline Tabagismo & $2(4,8)$ & $4(5,6)$ & $6(15,8)$ & $0,074^{*}$ \\
\hline Etilismo & $2(4,8)$ & $3(4,2)$ & $2(5,3)$ & $0,358^{*}$ \\
\hline Sedentarismo & $7(16,7)$ & $30(41,7)$ & $24(63,2)$ & $0,000^{*}$ \\
\hline Sono prejudicado & $20(47,6)$ & $32(44,4)$ & $16(42,1)$ & $0,620^{*}$ \\
Número de medicamentos** & $4,2 \pm 2,3$ & $4,0 \pm 2,4$ & $4,0 \pm 2,2$ & $0,889^{* *}$ \\
\hline
\end{tabular}

*Teste exato de Fisher; **ANOVA com ajuste de Bonferroni.

Tabela 3. Prevalência, Odds ratio e intervalo de confiança de $95 \%$ dos números de componentes da síndrome metabólica de acordo com o risco cardiovascular de Framingham dos idosos. Brasília, 2019. (n=154).

\begin{tabular}{|c|c|c|c|c|c|c|c|c|c|}
\hline \multirow{2}{*}{$\begin{array}{l}\text { N.o de CP } \\
N=154\end{array}$} & \multicolumn{3}{|c|}{ Baixo risco } & \multicolumn{3}{|c|}{ Moderado risco } & \multicolumn{3}{|c|}{ Elevado risco } \\
\hline & n (\%) & OR (IC 95\%) & Valor P & $\begin{array}{c}n \\
(\%)\end{array}$ & $\begin{array}{c}\text { OR } \\
\text { (IC 95\%) }\end{array}$ & Valor $\mathrm{P}$ & $\mathrm{n}(\%)$ & OR (IC 95\%) & Valor $\mathrm{P}$ \\
\hline $\begin{array}{c}1 \\
(n=6)\end{array}$ & $\begin{array}{c}3 \\
(7,1)\end{array}$ & $\begin{array}{c}2,79 \\
(0,54-14,43)\end{array}$ & 0,219 & $\begin{array}{c}3 \\
(4,2)\end{array}$ & $\begin{array}{c}1,14 \\
(0,22-5,85)\end{array}$ & 0,870 & - & - & \\
\hline $\begin{array}{c}2 \\
(n=39)\end{array}$ & $\begin{array}{c}14 \\
(33,3)\end{array}$ & $\begin{array}{c}2,30 \\
(1,06-4,98)\end{array}$ & 0,034 & $\begin{array}{c}22 \\
(30,6)\end{array}$ & $\begin{array}{c}1,68 \\
(0,80-3,49)\end{array}$ & 0.163 & $\begin{array}{c}3 \\
(7,5)\end{array}$ & $\begin{array}{c}0,17 \\
(0,05-0,60)\end{array}$ & 0,006 \\
\hline $\begin{array}{c}3 \\
(n=50)\end{array}$ & $\begin{array}{c}18 \\
(42,9)\end{array}$ & $\begin{array}{c}1,87 \\
(0,89-3,91)\end{array}$ & 0,094 & $\begin{array}{c}23 \\
(31,9)\end{array}$ & $\begin{array}{c}0,95 \\
(0,48-1,88)\end{array}$ & 0.896 & $\begin{array}{c}9 \\
(22,5)\end{array}$ & $\begin{array}{c}0,36 \\
(0,15-0,85)\end{array}$ & 0,020 \\
\hline $\begin{array}{c}4 \\
(n=40)\end{array}$ & $\begin{array}{c}6 \\
(14,3)\end{array}$ & $\begin{array}{c}0,38 \\
(0,14-0,99)\end{array}$ & 0,048 & $\begin{array}{c}15 \\
(20,8)\end{array}$ & $\begin{array}{c}0,60 \\
(0,28-1,25)\end{array}$ & 0.174 & $\begin{array}{c}19 \\
(47,5)\end{array}$ & $\begin{array}{c}4,01 \\
(1,83-8,74)\end{array}$ & 0,000 \\
\hline $\begin{array}{c}5 \\
(n=19)\end{array}$ & $\begin{array}{c}1 \\
(2,4)\end{array}$ & $\begin{array}{c}0,12 \\
(0,01-0,91)\end{array}$ & 0,048 & $\begin{array}{c}9 \\
(12,5)\end{array}$ & $\begin{array}{c}1,02 \\
(0,39-2,69)\end{array}$ & 0,954 & $\begin{array}{c}9 \\
(22,5)\end{array}$ & $\begin{array}{c}3,74 \\
(1,37-10,18)\end{array}$ & 0,009 \\
\hline \multicolumn{10}{|l|}{ SM } \\
\hline$\underset{(n=109)}{\operatorname{Sim}}$ & $\begin{array}{c}25 \\
(59,5)\end{array}$ & $\begin{array}{c}0,49 \\
(0,23-1,03)\end{array}$ & 0,062 & $\begin{array}{c}47 \\
(65,3)\end{array}$ & $\begin{array}{c}0,60 \\
(0,30-1,22)\end{array}$ & 0,161 & $\begin{array}{c}37 \\
(92,5)\end{array}$ & $\begin{array}{c}7,19 \\
(2,08-24,77)\end{array}$ & 0,001 \\
\hline $\begin{array}{c}\text { Não } \\
(n=45)\end{array}$ & $\begin{array}{c}17 \\
(40,5)\end{array}$ & - & & $\begin{array}{c}25 \\
(34,7)\end{array}$ & - & & $\begin{array}{c}3 \\
(7,5)\end{array}$ & - & \\
\hline
\end{tabular}

*CP: componente; SM: síndrome metabólica. 
Figura 1. Escore de Risco de Framingham de acordo com o estado nutricional e presença de síndrome metabólica em idosos. Brasília, 2019 ( $n=154) ; p=0,000$ (ANOVA com ajuste de Bonferroni).

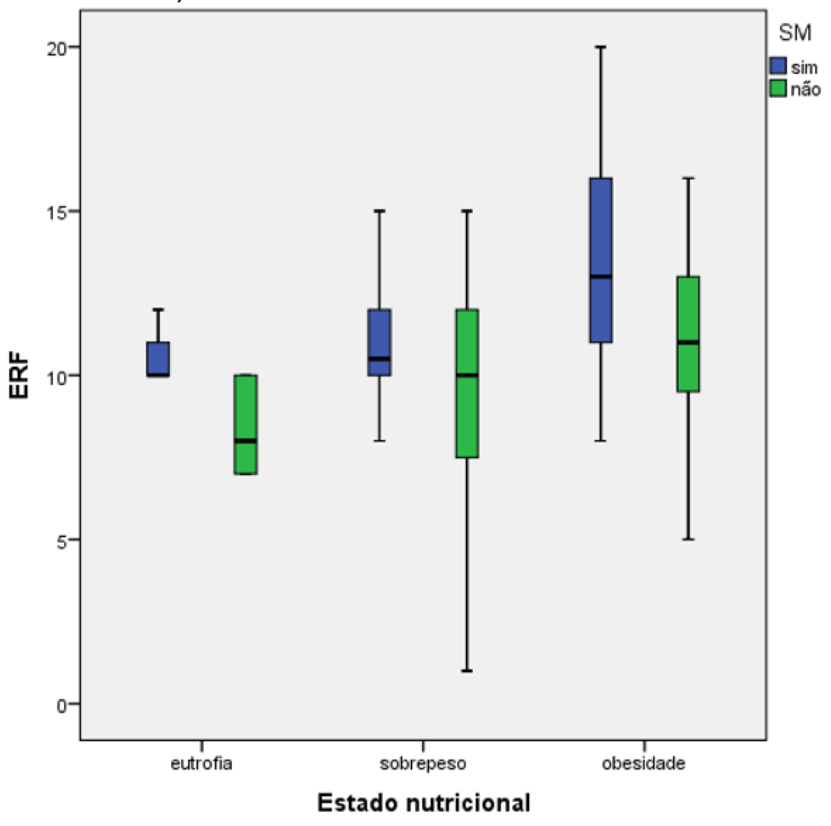

ERF: Escore de Risco de Framingham; SM: Síndrome Metabólica.

\section{DISCUSSÃO}

Neste estudo, observou-se que um elevado número de idosos hipertensos apresentou excesso de peso e SM. O número de idosos que apresentaram SM neste estudo foi considerado elevado, uma vez que outras pesquisas evidenciaram prevalências inferiores. No Brasil, uma prevalência de 29,2\% foi encontrada em idosos institucionalizados em Natal-Rio Grande do Norte, ${ }^{6}$ $42 \%$ em hipertensos, predominantemente idosos residentes em Juiz de Fora-Minas Gerais, ${ }^{7} 45,2 \%$ em idosos de Niterói-Rio de Janeiro, ${ }^{8} 51 \%$ em idosos de uma Unidade de Referência de Reabilitação Física de Belém-Pará ${ }^{9}$ e 58,65\% em idosos usuários do sistema único de saúde da cidade de Goiânia-Goiás. ${ }^{10}$ Em estudos internacionais, as prevalências variaram entre 45,9\% e $72,9 \% .{ }^{16-19}$ Cabe ressaltar um estudo conduzido no Egito, com hipertensos, no qual encontraram uma prevalência de SM de $76,6 \%,{ }^{16}$ semelhante à observada neste estudo.

Sabe-se que alguns fatores podem influenciar na SM, a exemplo da obesidade. Os indivíduos obesos são mais propensos ao diagnóstico de SM. ${ }^{20}$ Além disso, a obesidade é fator de risco independente para o desenvolvimento de hipertensão arterial sistêmica, dislipidemias e maior resistência insulínica. Essas patologias agregam os componentes que caracterizam a SM, e, por isso, indivíduos obesos são mais predispostos a desenvolverem a SM. ${ }^{21}$ Essa elevada prevalência de SM nessas populações geralmente é decorrente da exposição contínua aos vários fatores de risco que predispõe ao quadro, sendo eles o consumo de uma dieta inadequada, tabagismo, etilismo e sedentarismo, que levam direta ou indiretamente a um quadro de excesso de peso e consequente maior RCV. ${ }^{22}$

Com a estratificação do RCV, os resultados deste estudo demonstraram que a maioria dos idosos tem um moderado risco de desenvolver algum evento cardiovascular nos próximos dez anos, de acordo com o Framingham. As variáveis, sexo, idade, sedentarismo, parâmetros bioquímicas e DM foram relacionadas ao RCV neste estudo.

A maioria dos estudos conduzidos com o objetivo de analisar o RCV evidenciou uma maior prevalência de baixo RCV. Em uma amostra de brasileiros hipertensos, predominantemente idosa, foi observado que $74 \%$ apresentaram baixo, $14 \%$ moderado e $12 \%$ elevado RCV. ${ }^{7}$ Neste mesmo sentido, um estudo realizado com uma população adulta nigeriana verificou que $86,3 \%$ possuíam baixo, 11,8\% moderado e 1,9\% elevado RCV. ${ }^{23}$ Em um estudo conduzido no Irã, com adultos diagnosticados com SM, 77,5\% possuíam baixo, $16,3 \%$ moderado e $6,3 \%$ elevado $\mathrm{RCV} .{ }^{14}$ Por outro lado, $48 \%$ da população egípcia idosa apresentou de moderado a elevado $\mathrm{RCV},{ }^{16}$ corroborando com os resultados evidenciados neste estudo.

Foi demonstrado que ser do sexo feminino influencia significativamente no RCV. Essa predominância pode ser justificada ao fato da mulher apresentar maior sobrevida em relação ao sexo masculino, além da forte relação entre pressão arterial e menopausa, devido à menor produção de hormônios femininos, como o estrogênio, ocasionando o aumento dos níveis pressóricos, ${ }^{24}$ e ainda ao maior acúmulo de gordura abdominal no período pós-menopausa. ${ }^{25}$

Outro fato observado neste estudo foi que a média de idade aumenta de acordo com a gravidade do RCV. Em um estudo de base populacional realizado no Paraná, foi analisada a simultaneidade de fatores de RCV e observado que $95,9 \%$ dos idosos tinham dois ou mais fatores de risco. Sabe-se que a idade avançada é considerada um fator de risco cardiovascular não modificável que, quando somado com aqueles modificáveis, aumenta a possibilidade de ocorrência de doença cardiovascular. ${ }^{26}$

Elevados níveis pressóricos também foram evidenciados naqueles com elevado RCV. No Brasil, a HAS atinge 32,5\% de indivíduos adultos e mais de $60 \%$ dos idosos, contribuindo direta ou indiretamente para 50\% das mortes por DCV. Em 2013, 29,8\% dos óbitos foram decorrentes de DCV, a principal causa de morte no país. As DCV são ainda responsáveis por alta frequência de internações, com custos socioeconômicos elevados. Há uma associação direta e linear entre envelhecimento e prevalência da HAS, relacionada ao aumento da expectativa de vida da população brasileira e ao aumento na população de idosos na última década. ${ }^{1}$

Em relação ao sedentarismo, outros estudos também reforçam sua influência negativa no $\mathrm{RCV}^{27-29}$ É evidente que mudanças no estilo de vida apresentam efeito positivo na redução do ERF, diminuindo a chance dos indivíduos sofrerem eventos coronarianos nos próximos dez anos..$^{30}$

Neste estudo, os idosos com elevado RCV apresentaram um pior perfil bioquímico, principalmente no que tange às 
dislipidemias e ao controle glicêmico. Idosos hipertensos, diabéticos e com dislipidemias apresentam maiores fatores de risco para ocorrência de DCV. ${ }^{21}$ Outros estudos também evidenciaram que as dislipidemias aumentam significativamente o RCV. ${ }^{13,31,32}$

Indivíduos que apresentam maior RCV possuem mais chances de desenvolver DM tipo 2, e aqueles que já possuem o diagnóstico de DM podem agravar o quadro de SM. ${ }^{33}$ Cabe ressaltar que neste estudo $84,2 \%$ dos idosos que apresentaram elevado RCV tinham DM. Ainda, as DCV são a principal causa de óbito entre as pessoas com DM, sendo responsáveis por aproximadamente metade dos óbitos por DM na maioria dos países. ${ }^{4}$ Existe, ainda, uma forte relação entre a SM e o desenvolvimento do DM, que se intensifica quanto maior o número de componentes da SM. ${ }^{21}$ A HAS, isoladamente, já confere um risco cardiometabólico, que pode prejudicar ainda mais a resistência à insulina no metabolismo dos indivíduos com SM. ${ }^{34}$

Os resultados deste estudo evidenciaram que os idosos hipertensos com SM e os que são obesos apresentaram maiores ERF. A SM aumentou significativamente a chance do idoso apresentar elevado RCV. Idosos obesos apresentaram elevado RCV, fato também observado em outro estudo. ${ }^{35} \mathrm{~A} \mathrm{SM}$ aumenta o RCV por meio de uma cadeia de eventos que envolvem hiperglicemia, vasoconstrição periférica, retenção de sódio, maior produção de TG e LDL, aterosclerose, quadro pró-trombótico e pró-inflamatório sistêmico, que se iniciam por meio da resistência insulínica gerada pela obesidade. ${ }^{21}$ Sabe-se que a presença de pelo menos três componentes da SM aumenta a mortalidade cardiovascular em cerca de 2,5 vezes. ${ }^{36}$

Este estudo demonstrou, ainda, que a SM exerce uma influência significativa no RCV, resultado corroborado por outros estudos. ${ }^{16,23,35,37-39}$ Além disso, a prevalência de moderado e elevado RCV foi estatisticamente mais evidente nos idosos com $\mathrm{SM}$, assim como o número de componentes influenciou no RCV, resultado similar de outras pesquisas. ${ }^{13,14,38}$

Uma pesquisa conduzida na Itália avaliou o desfecho cardiovascular de 1.191 idosos hipertensos, sendo que $48,5 \%$ apresentavam SM, observando que, durante dez anos de acompanhamento, 39 acidentes vasculares cerebrais e 120 eventos coronários ocorreram. Os autores concluíram que a SM foi relacionada ao RCV, principalmente nos idosos com DM, parâmetros da pressão arterial e glicemia comprometidos. ${ }^{40}$

A SM e o RCV são em grande parte evitáveis. Exercícios regulares, dieta saudável e controle de peso são recomendações para modificar o estilo de vida, uma vez que envolvem mudanças comportamentais. ${ }^{41}$ Um ensaio clínico randomizado realizado na China, com idosos, objetivou avaliar os efeitos de longo prazo de uma intervenção comunitária no estilo de vida sobre os indicadores bioquímicos e na prevalência de SM em 69 idosos, demonstrando que exercício físico e intervenções na dieta diminuíram os indicadores e a prevalência da SM. ${ }^{42}$

Neste contexto, sabe-se que é necessária uma abordagem multidisciplinar em saúde, porém a SM pode ser identificada rotineiramente pelos enfermeiros da atenção primária à saúde. Ao conhecer as possibilidades de critérios de diagnóstico da SM e os fatores envolvidos nessa desordem, o enfermeiro tem um importante papel no que tange ao diagnóstico, ao planejamento de cuidados e à prevenção da SM.

Isto posto, o profissional de enfermagem tem uma importante atuação no sentido de promover saúde e capacitar os idosos para se tornarem responsáveis pela sua saúde. Pacientes com DCNT requerem estratégias de cuidado especiais. Os profissionais de enfermagem devem garantir que esses pacientes recebam informações, orientações e acompanhamento adequado, a fim de adotarem comportamentos que previnam o surgimento de outras condições crônicas, principalmente a SM.

Neste estudo, algumas limitações também devem ser apontadas, especialmente em relação ao método, por se tratar de um estudo transversal que não permite estabelecer relações de causalidade. Ainda, acredita-se que possam existir vieses que não foram controlados na seleção dos participantes, uma vez que a obesidade foi uma variável presente na maioria dos participantes e estes pacientes apresentam maior risco de desenvolver SM. Sugere-se, portanto, que estudos futuros sejam realizados considerando esse critério na seleção da amostra e a realização de análise multivariada. Apesar disso, acredita-se que os resultados deste estudo podem contribuir para geração de conhecimento científico nessa temática.

\section{CONSIDERAÇÕES FINAIS E IMPLICAÇÕES PARA A PRÁTICA}

Os resultados deste estudo mostraram que os idosos hipertensos apresentaram uma elevada prevalência de SM, o que aumentou significativamente o RCV. O sexo feminino, a idade avançada, o sedentarismo, o DM, os parâmetros clínicos e bioquímicos influenciaram negativamente o RCV em idosos hipertensos. Identificar esses fatores possibilita um melhor planejamento da assistência de enfermagem dos profissionais que atuam na atenção primária à saúde.

É importante reforçar que o RCV e a SM são preveníveis; portanto, os profissionais da atenção primária à saúde devem atuar ativamente, a fim de implementar medidas preventivas eficazes. A atenção primaria é a porta de entrada dos cuidados da população, o local onde ocorre a prevenção, o diagnóstico e os cuidados do paciente. Assim, se o cuidado for eficaz, a ponto de identificar os idosos com elevado RCV e com diagnóstico de $\mathrm{SM}$, os profissionais de enfermagem podem elaborar planos para cada paciente ter um estilo de vida mais saudável e ensiná-los a ter controle da saúde. A partir dos resultados obtidos, sugere-se que estudos semelhantes sejam realizados periodicamente com o objetivo de identificar os fatores envolvidos nessas condições crônicas na população idosa.

\section{FINANCIAMENTO}

Fundação de Amparo à Pesquisa do Distrito Federal (FAP-DF), Brasília, Brasil. Edital n.ำ03/2016. 


\section{CONTRIBUIÇÕES DOS AUTORES}

Desenho do estudo. Aquisição, análise de dados e interpretação dos resultados. Redação e revisão crítica do manuscrito. Aprovação da versão final do artigo. Responsabilidade por todos os aspectos do conteúdo e a integridade do artigo publicado. Manoela Vieira Gomes da Costa. Marina Morato Stival.

Análise de dados e interpretação dos resultados. Redação e revisão crítica do manuscrito. Aprovação da versão final do artigo. Responsabilidade por todos os aspectos do conteúdo e a integridade do artigo publicado. Luciano Ramos de Lima. Izabel Cristina Rodrigues da Silva. Tania Cristina Morais Santa Barbara Rehem. Silvana Schwerz Funghetto.

\section{EDITOR ASSOCIADO}

\section{Gerson Luiz Marinho}

\section{REFERÊNCIAS}

1. Malachias M, Plavnik FL, Machado CA, Malta D, Scala LCN, Fuchs S 7a Diretriz Brasileira de Hipertensão Arterial: Capítulo 1 - Conceituação, Epidemiologia e Prevenção Primária. Arq Bras Cardiol. 2016;107(3, Suppl):1-6. http://dx.doi.org/10.5935/abc.20160151. PMid:27819380

2. Ministério da Saúde (BR). Estratégias para o cuidado da pessoa com doença crônica: hipertensão arterial sistêmica [Internet]. Brasília: Ministério da Saúde; 2013 (Cadernos de Atenção Básica, 37). [citado 2019 Dez 5]. Disponível em: http://bvsms.saude.gov.br/bvs/publicacoes/ estrategias_cuidado_pessoa_doenca_cronica.pdf

3. Moreira AACM, Costa EFA, Miranda RD, Wajngarten M, Galera SC Alencar Filho AC et al. Updated Geriatric Cardiology Guidelines of the Brazilian Society of Cardiology - 2019. Arq Bras Cardiol. 2019;112(5):649705. http://dx.doi.org/10.5935/abc.20190086. PMid:31188969.

4. Oliveira JEP, Vencio S. Diretrizes da Sociedade Brasileira de Diabetes 2017-2018 [Internet]. São Paulo: Ed Clannad; 2017 [citado 2019 Dez 5]. Disponível em: https://www.diabetes.org.br/profissionais/images/2017/ diretrizes/diretrizes-sbd-2017-2018.pdf

5. Félix NDC, Nóbrega MMLD. Síndrome metabólica: análise conceitual no contexto da enfermagem. Rev Lat Am Enfermagem. 2019;27:e3154. http://dx.doi.org/10.1590/1518-8345.3008.3154. PMid:31432914.

6. Sales MC, Oliveira LP, Liberalino LCP, Cunha ATO, Sousa SES, Lemos TMAM et al. Frequency of metabolic syndrome and associated factors in institutionalized elderly individuals. Clin Interv Aging. 2018 nov; 13:245364. http://dx.doi.org/10.2147/CIA.S177731. PMid:30555225.

7. Paula EA, Paula RB, Costa DMN, Colugnati FAB, Paiva EP. Cardiovascular risk assessment in hypertensive patients. Rev Lat Am Enfermagem. 2013 jun;21(3):820-7. http://dx.doi.org/10.1590/S0104-11692013000300023. PMid:23918030.

8. Saad MAN, Cardoso GP, Martins WA, Velarde LGC, Cruz RA Fo. Prevalence of metabolic syndrome in elderly and agreement among four diagnostic criteria. Arq Bras Cardiol. 2014;102(3):263-9. http:// dx.doi.org/10.5935/abc.20140013. PMid:24676226.

9. Calixto SCS, Vinagre RMFD, Rocha GF, França TG. Prevalência da Síndrome Metabólica em Idosos. Saúde em Foco. 2017;3(2):119-35. http://dx.doi.org/10.12819/rsf.2016.3.2.9.

10. Vieira EC, Peixoto MR, Silveira EA. Prevalence and factors associated with Metabolic Syndrome in elderly users of the Unified Health System. Rev Bras Epidemiol. 2014 dez;17(4):805-17. http://dx.doi.org/10.1590/18094503201400040001. PMid:25388482.

11. Silva JF, Freire JAP, Frota KMG, Júnior AJS. Fatores de risco cardiovascular e prevalência de síndrome metabólica em idosos. Rev Bras em promoção da Saúde. 2014 dez;27(4):477-84. http://dx.doi. org/10.5020/18061230.2014.p477

12. van Herpt TTW, Dehghan A, van Hoek M, Ikram MA, Hofman A, Sijbrands EJG et al. The clinical value of metabolic syndrome and risks of cardiometabolic events and mortality in the elderly: The Rotterdam study. Cardiovasc Diabetol. 2016 dez 27;15(1):69. http://dx.doi.org/10.1186/ s12933-016-0387-4. PMid:27117940.

13. LiW, Song F, Wang X, Wang D, Chen D, Yue W et al. Relationship between metabolic syndrome and its components and cardiovascular disease in middle-aged and elderly Chinese population: a national cross-sectional survey. BMJ Open. 2019 ago;9(8):e027545. http://dx.doi.org/10.1136/ bmjopen-2018-027545. PMid:31427317.

14. Jahangiry L, Farhangi MA, Rezaei F. Framingham risk score for estimation of 10-years of cardiovascular diseases risk in patients with metabolic syndrome. J Health Popul Nutr. 2017 nov;36(1):36. http:// dx.doi.org/10.1186/s41043-017-0114-0. PMid:29132438.

15. Mastroeni MF, Mastroeni SSBS, Erzinger GS, Marucci MFN. Antropometria de idosos residentes no município de Joinville-SC, Brasil. Rev Bras Geriatr Gerontol. 2010 abr;13(1):29-40. http://dx.doi.org/10.1590/ S1809-98232010000100004.

16. Abd Elaziz KM, Gabal MS, Aldafrawy OA, Abou Seif HA-A, Allam MF Prevalence of metabolic syndrome and cardiovascular risk factors among voluntary screened middle-aged and elderly Egyptians. J Public Health (Oxf). 2015 dez;37(4):612-7. http://dx.doi.org/10.1093/pubmed/ fdu097. PMid:25503580.

17. Dussaillant C, Echeverría G, Villarroel L, Yu C, Rigotti A, Marín P. Metabolic syndrome prevalence is not associated with diet quality in the Chilean elderly population: a cross sectional analysis from the National Health Survey 2009-2010. J Aging Res Clin Pract. 2016;5:132-8. http://dx.doi. org/10.14283/jarcp.2016.104.

18. Pradeepa R, Surendar J, Indulekha K, Chella S, Anjana RM, Mohan V Prevalence of metabolic syndrome and its association with coronary artery disease among an urban elderly south Indian population (CURES-145). J Assoc Physicians India [Internet]. 2016 [cited 2020 Feb 15];64(5):20-5 Available from: http://www.japi.org/may_2016/03_oa_prevalence_of_ metabolic.pdf

19. Ortiz-Rodríguez MA, Yáñez-Velasco L, Carnevale A, Romero-Hidalgo S, Bernal D, Aguilar-Salinas C et al. Prevalence of metabolic syndrome among elderly Mexicans. Arch Gerontol Geriatr. 2017 nov;73:288-93. http://dx.doi.org/10.1016/j.archger.2017.09.001. PMid:28910752.

20. França SL, Lima SS, Vieira JRDS. Metabolic syndrome and associated factors in adults of the amazon region. PLoS One. 2016 dec;11(12):114. http://dx.doi.org/10.1371/journal.pone.0167320

21. Simão AF, Precoma DB, Andrade JP, Correa Filho H, Saraiva JFK, Oliveira GMM et al. I Diretriz brasileira de prevenção cardiovascular. Arq Bras Cardiol. 2013;101(6):1-63. http://dx.doi.org/10.5935/abc.2013S012. PMid:24554026.

22. Silva PAB, Sacramento AJ, Carmo CID, Silva LB, Silqueira SMF, Soares SM. Fatores associados à síndrome metabólica em idosos: estudo de base populacional. Rev Bras Enferm. 2019;72(2 Suppl):221-8. http:// dx.doi.org/10.1590/0034-7167-2018-0620. PMid:31826214.

23. Oguoma VM, Nwose EU, Skinner TC, Richards RS, Digban KA, Onyia IC. Association between metabolic syndrome and 10-year risk of developing cardiovascular disease in a Nigerian population. Int Health. 2016 set;8(5):354-9. http://dx.doi.org/10.1093/inthealth/ihw013. PMid:27118483.

24. Santos LB, Lima WL, Souza JMO, Magro MCS, Duarte TTP. Risco cardiovascular em usuários hipertensos da atenção primária à saúde. Rev enferm UFPE line. 2018;12(5):1303-9. http://dx.doi.org/10.5205/19818963-v12i5a234979p1303-1309-2018

25. Fogaça EM, Theodoro H, Mendes KG, Olinto MTA. Prevalência de obesidade em mulheres na pós-menopausa atendidas em um ambulatório no sul do Brasil. Rev da Assoc Bras Nutr [Internet]. 2019 [cited 2020 Jan 10];10(1):46-52. Available from: https://www.rasbran. com.br/rasbran/article/view/663

26. D'Amico MM, de Souza RKT. Simultaneidade de fatores de risco cardiovascular controláveis: estudo de base populacional. Rev Bras Cardiol [Internet]. 2014 [cited 2020 Jan 10];27(5):318-26. Available from: http://www.onlineijcs.org/english/sumario/27/pdf/v27n5a05.pdf

27. Young DR, Hivert M-F, Alhassan S, Camhi SM, Ferguson JF, Katzmarzyk PT et al. Sedentary behavior and cardiovascular morbidity and mortality: A science advisory from the american heart association. 
Circulation. 2016 set 27;134(13):e262-79. http://dx.doi.org/10.1161/ CIR.0000000000000440. PMid:27528691.

28. Lavie CJ, Ozemek C, Carbone S, Katzmarzyk PT, Blair SN. Sedentary Behavior, Exercise, and Cardiovascular Health. Circ Res. 2019 mar;124(5):799-815. http://dx.doi.org/10.1161/CIRCRESAHA.118.312669. PMid:30817262.

29. Leiva AM, Martínez MA, Cristi-Montero C, Salas C, Ramírez-Campillo $R$, Díaz Martínez $X$ et al. El sedentarismo se asocia a un incremento de factores de riesgo cardiovascular y metabólicos independiente de los niveles de actividad física. Rev Med Chil. 2017 abr;145(4):458-67. http://dx.doi.org/10.4067/S0034-98872017000400006. PMid:28748993.

30. Soares TS, Piovesan CH, Gustavo AS, Macagnan FE, Bodanese LC, Feoli AMP. Alimentary habits, physical activity, and framingham global risk score in metabolic syndrome. Arq Bras Cardiol. 2014;102(4):37482. http://dx.doi.org/10.5935/abc.20140029. PMid:24652053.

31. Ormazabal V, Nair S, Elfeky O, Aguayo C, Salomon C, Zuñiga FA. Association between insulin resistance and the development of cardiovascular disease. Cardiovasc Diabetol. 2018 dez 31;17(1):122. http://dx.doi.org/10.1186/s12933-018-0762-4. PMid:30170598.

32. Kopin L, Lowenstein CJ. Dyslipidemia. Ann Intern Med. 2017 dez 5;167(11):ITC81-96. http://dx.doi.org/10.7326/AITC201712050. PMid:29204622.

33. Gurka MJ, Filipp SL, Pearson TA, DeBoer MD. Assessing baseline and temporal changes in cardiometabolic risk using metabolic syndrome severity and common risk scores. J Am Heart Assoc. 2018 ago 21;7(16):e009754. http://dx.doi.org/10.1161/JAHA.118.009754. PMid:30369320.

34. Faulkner JL, Belin de Chantemèle EJ. Sex hormones, aging and cardiometabolic syndrome. Biol Sex Differ. 2019 dez 1;10(1):30. http:// dx.doi.org/10.1186/s13293-019-0246-6. PMid:31262349.

35. Arnlöv J, Ingelsson E, Sundström J, Lind L. Impact of body mass index and the metabolic syndrome on the risk of cardiovascular disease and death in middle-aged men. Circulation. 2010 jan 19;121(2):230-6. http:// dx.doi.org/10.1161/CIRCULATIONAHA.109.887521. PMid:20038741.

36. Carvalho MHC. I Diretriz Brasileira de Diagnóstico e Tratamento da Síndrome Metabólica. Arq Bras Cardiol. 2005 abr;84:3-28. http://dx.doi. org/10.1590/S0066-782X2005000700001.

37. Motamed N, Rabiee B, Roozafzai F, Zamani F, Faraji AH, Maadi $M$ et al. Metabolic syndrome and cardiovascular risk assessment tools estimations of 10-year cardiovascular risk: A population-based study. Acta Cardiol. 2018 set 3;73(5):439-46. http://dx.doi.org/10.1080/0001 5385.2017.1410335. PMid:29188761.

38. Yang W, Ma R, Zhang X, Guo H, He J, Mao L et al. Comparison between metabolic syndrome and the framingham risk score as predictors of cardiovascular diseases among Kazakhs in Xinjiang. Sci Rep. 2018 dez 7;8(1):16474. http://dx.doi.org/10.1038/s41598-018-34587-1. PMid:30405180.

39. Pinho PM, Machado LMM, Torres RS, Carmin SEM, Mendes WAA, Silva ACM et al. Síndrome metabólica e sua relação com escores de risco cardiovascular em adultos com doenças crônicas não transmissíveis. Rev Soc Bras Clín Méd [Internet]. 2014 [citado 2020 Feb 2];12(1):2230. Disponível em: http://files.bvs.br/upload/S/1679-1010/2014/v12n1/ a4030.pdf\%60

40. Pierdomenico SD, Pierdomenico AM, Di Tommaso R, Coccina F, Di Carlo S, Cuccurullo F et al. Metabolic Syndrome and Cardiovascular Risk in Elderly Treated Hypertensive Patients. Am J Hypertens. 2015 jul 29;29(3):hpv121. http://dx.doi.org/10.1093/ajh/hpv121.PMid:26224400.

41. Aghakhanian F, Wong C, Tan JSY, Yeo LF, Ramadas A, Edo J et al Metabolic syndrome and cardiometabolic risk factors among indigenous Malaysians. Public Health. 2019 nov; 176:106-13. http://dx.doi.org/10.1016/j. puhe.2018.10.001. PMid:30509859.

42. Chang $\mathrm{S}-\mathrm{H}, \mathrm{Chien} \mathrm{N}-\mathrm{H}, \mathrm{Yu} \mathrm{C}-\mathrm{Y}$. Long-term lifestyle intervention in elderly with metabolic syndrome. Clin Nurs Res. 2019 jul 24;28(6):658-75. http://dx.doi.org/10.1177/1054773817749923. PMid:29276844. 